\title{
Sensitivity Enhancement in Evanescent Optical Waveguide Sensors
}

\author{
G. J. Veldhuis, O. Parriaux, H. J. W. M. Hoekstra, and P. V. Lambeck
}

\begin{abstract}
It is shown, that the sensitivity of the effective refractive index on the cladding index in evanescent optical waveguide sensors, can be larger than unity. This implies that the attenuation of a guided wave propagating in a waveguide immersed in an absorptive medium can be made larger than that of a free-space wave propagating through the same medium. The conditions and physical explanation for this puzzling behavior are identified and as a practical application, an absorption sensor, based on a suspended silicon slab waveguide, is proposed where the sensitivity is enhanced by a factor of 1.35 .
\end{abstract}

Index Terms-Chemical sensing, evanescent field sensing, integrated optics, refractometry.

\section{INTRODUCTION}

C HEMICAL sensing is one of the noncommunication application fields where integrated optics can play an important role. In general, measurand-induced changes of refractive, absorptive or luminescent properties of a medium or of a layer located in the evanescent wave region of the propagating mode are measured. In this paper, we will focus on refractive and absorptive sensors where the sensing operation consists of the measurement of changes of the real or imaginary part of the effective refractive index caused by measurand-induced changes of the refractive index of the cladding. This has so far been made by using schemes such as Mach-Zehnder interferometers [1], [2], grating couplers [3]-[5], bent waveguides [6] or absorbing waveguides [7]. All these devices are essentially based on a three-layer slab waveguide configuration.

In a free-space beam sensing scheme, discrete optical components such as mirrors, lenses, and spatial filters are used to define the wave front and the trajectory of the beam. The integrated optic approach allows for a significant simplification of the optical system, since the light routing and processing can be accomplished by a waveguide circuit. Nevertheless, this approach appears to have a drawback. It is generally assumed that the sensitivity of a guided wave to changes of the cladding index is smaller or much smaller than that of a free-space beam. This assumption rests on the intuitive representation that only part of the optical field guided in the

Manuscript received April 8, 1999; revised November 29, 1999. This work was supported by the Dutch Innovative Research Program Electro-Optics.

H. J. W. M. Hoekstra and P. V. Lambeck are with the Mesa Research Institute, University of Twente, 7500 AE Enschede, The Netherlands.

G. J. Veldhuis was with Mesa Research Institute, University of Twente, 7500 AE Enschede, The Netherlands. He is now with Philips Research, Eindhoven 5656 AA, The Netherlands.

O. Parriaux is with the Laboratoire TSI, Université Jean Monnet, Saint-Etienne F-42023, France.

Publisher Item Identifier S 0733-8724(00)03983-9. waveguide "sees" the analyzed medium. Here the sensitivity is defined as the ratio of the effective index change of the guided mode to the refractive index change of the core layer environment, i.e., both cladding and substrate. This ratio is 1 for a free-space beam.

Nevertheless, for practical application the sensitivity should be as high as possible and therefore the waveguide parameters should be chosen with care. In [4], analytical expressions for the sensitivity of a given slab waveguide for both homogeneous and surface sensing were derived from the characteristic equation for a three-layer slab waveguide. This allows a case-by-case optimization of the waveguide parameters. We showed [8] that the maximum obtainable sensitivity for a a three-layer slab waveguide is only dependent on two normalized optogeometric parameters which enabled us to give the optimization conditions once for all cases and to present universal graphs of the maximum obtainable sensitivity.

In this paper we report the peculiar effect that for TM polarization and strong guidance conditions the sensitivity, with respect to changes of both the real and imaginary part of the environmental index, can indeed be larger than unity. This means that an integrated optical read-out and sensing circuit can be made with an even higher sensitivity than in a free-space configuration. This also implies that the attenuation of a guided wave propagating in a waveguide embedded in an absorptive medium can be larger than that of a free-space beam propagating in the said medium. To the knowledge of the authors, this phenomenon was not previously reported.

In Section II, the conditions for the sensitivity enhancement are identified by means of an analysis in terms of normalized parameters similar to the treatment of [8]. It will be shown that the effect only occurs for transverse magnetic (TM) polarization. The effect is treated in detail for a symmetric waveguide $\left(n_{s}=n_{c}\right)$. Section III presents universal graphs which show the dependence of the effect on the waveguide contrast and proposes a realistic sensor based on a suspended silicon waveguide technology with a sensitivity of 1.35, i.e., exhibiting absorption or gain enhancement. Finally, Section IV gives a physical explanation of the enhancement effect.

\section{CONDITIONS FOR SENSITIVITY ENHANCEMENT}

First, normalized expressions for the sensitivity will be derived which will be used to investigate the occurrence and magnitude of the sensitivity enhancement. The starting point for the 
analysis is the well-known characteristic equation for a threelayer slab waveguide as illustrated in Fig. 1 [10]

$$
\begin{array}{r}
\frac{2 \pi t}{\lambda} \sqrt{n_{f}^{2}-N^{2}}-\arctan \left[\sqrt{\frac{N^{2}-n_{s}^{2}}{n_{f}^{2}-N^{2}}}\left(\frac{n_{f}}{n_{s}}\right)^{2 p}\right] \\
-\arctan \left[\sqrt{\frac{N^{2}-n_{c}^{2}}{n_{f}^{2}-N^{2}}}\left(\frac{n_{f}}{n_{c}}\right)^{2 p}\right]-m \pi=0
\end{array}
$$

where $t$ is the thickness of the waveguide core layer, $n_{s}, n_{f}$ and $n_{c}$ are the refractive indices of the substrate, core and cover layer respectively, $N$ is the effective refractive index, $m$ is an integer defining the mode order, $p=0$ for TE- and $p=1$ for TM-polarization while $\lambda$ is the free-space wavelength. Equation (1) can be rewritten in a more compact normalized form as (2), shown at the bottom of the page, where $\alpha_{s}, \alpha_{c}, X$ and $T$ are normalized dimensionless parameters, defined as

$$
\begin{gathered}
\alpha_{s}=1-\left(\frac{n_{s}}{n_{f}}\right)^{2} \\
\alpha_{c}=1-\left(\frac{n_{c}}{n_{f}}\right)^{2} \\
X=1-\left(\frac{N}{n_{f}}\right)^{2} \\
T=\frac{2 \pi n_{f} t}{\lambda} .
\end{gathered}
$$

Here, $\alpha_{s}$ and $\alpha_{c}$ represent the normalized refractive index contrasts at the substrate and cover side respectively, $X$ is the normalized effective refractive index and $T$ is the normalized waveguide thickness. Note (from (2)), that for fixed polarization and mode-order, $T=T\left(X, \alpha_{s}, \alpha_{c}\right)$.

The sensitivity to changes in the cover can be found by differentiating (1)

$$
\begin{aligned}
S & \equiv \frac{\partial N}{\partial n_{c}}=\frac{P_{c}}{P_{\text {tot }}}\left[\sqrt{\frac{1-\alpha_{c}}{1-X}}+2 p\left(\sqrt{\frac{1-X}{1-\alpha_{c}}}-\sqrt{\frac{1-\alpha_{c}}{1-X}}\right)\right] \\
& =\frac{N}{n_{c}}\left\{1+\frac{N^{2}-n_{c}^{2}}{N^{2}}\right\} \frac{P_{c}}{P_{\text {tot }}}
\end{aligned}
$$

where we have used (3)-(5) and $P_{c} / P_{\text {tot }}$ is the fraction of modal power located in the cover layer [4]

$$
\frac{P_{c}}{P_{\mathrm{tot}}}=\frac{Q_{c}}{T+Q_{s}+Q_{c}} \frac{X}{\alpha_{c}}
$$

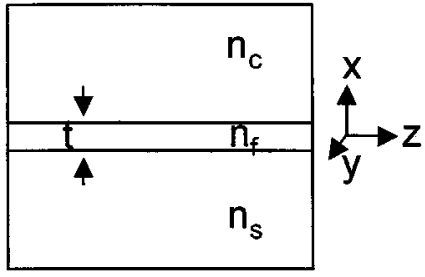

Fig. 1. Schematic representation of the slab waveguide under study. The grey area indicates the normal electric field component of the $\mathrm{TM}_{0}$ mode in a large-guidance waveguide.

with the normalized penetration depths [10]

$$
Q_{i}=\frac{1}{\sqrt{\alpha_{i}-X}} \cdot\left[\frac{1-\alpha_{i}}{1-X \cdot\left(2-\alpha_{i}\right)}\right]^{p} \quad i=s, c .
$$

Note (from (7)), that for fixed polarization and mode-order, also $S=S\left(X, \alpha_{s}, \alpha_{c}\right)$.

For both polarizations, the sensitivity at the limits of the variation domain of $X$ shows a similar behavior. For a very thick waveguide $(X \rightarrow 0)$ it is clear that $P_{c} / P_{\text {tot }} \rightarrow 0$ and therefore $S \rightarrow 0$. At cutoff, three different cases can be distinguished. If the waveguide is symmetric $\left(\alpha \equiv \alpha_{c}\right.$ and $\left.X \rightarrow \alpha\right)$, then $S \rightarrow P_{c} / P_{\text {tot }} \rightarrow 0.5$. If $n_{c}>n_{s}\left(X \rightarrow \alpha_{c}\right)$ then $S \rightarrow P_{c} / P_{\text {tot }} \rightarrow 1$. And finally, for $n_{c}<n_{s}\left(X \rightarrow \alpha_{s}\right)$ it turns out that $S \rightarrow 0$ since in that case $P_{c} / P_{\text {tot }} \rightarrow 0$.

Since by definition $\alpha_{c}>X$, it can immediately be seen from (7) that for transverse electric (TE) polarization, where $p=0$, $S_{\mathrm{TE}}<P_{c} / P_{\text {tot }}<1$ in all cases. However, for TM polarization, where $p=1$, a second term is present in the sensitivity expression (7) and it cannot be excluded a priori that the term between brackets will be larger than 1 and that this also can lead to $S_{\mathrm{TM}}>P_{c} / P_{\text {tot. }}$. In the following it will be investigated whether conditions exist for this to occur.

For given refractive indices of the layers and a given wavelength, we can find the condition (i.e., thickness) for maximum sensitivity to changes in the cover by solving

$$
\frac{\partial}{\partial t} \frac{\partial N}{\partial n_{c}}=0 .
$$

The mathematics for solving this equation can be simplified by considering

$$
\frac{\partial}{\partial t} \frac{\partial N}{\partial n_{c}}=\frac{\partial S}{\partial t}=\frac{\partial S}{\partial X} \frac{\partial X}{\partial t} .
$$

Since $\delta X / \delta t \neq 0$, the condition for maximum sensitivity is obtained by solving $\delta S / \delta X=0$. After some algebraic calculations, the exact condition for maximum sensitivity can be found as

$$
1+3 \frac{1-X}{X}+Q_{c}-p \frac{4(1-X)}{1+\alpha_{c}-2 X}-\frac{Y_{s} Q_{s}+Y_{c} Q_{c}}{T+Q_{s}+Q_{c}}=0
$$

$$
T=\frac{\arctan \left[\sqrt{\frac{a_{s}-X}{X}}\left(\frac{1}{1-\alpha_{s}}\right)^{p}\right]+\arctan \left[\sqrt{\frac{\alpha_{c}-X}{X}}\left(\frac{1}{1-\alpha_{c}}\right)^{p}\right]+m \pi}{\sqrt{X}}
$$




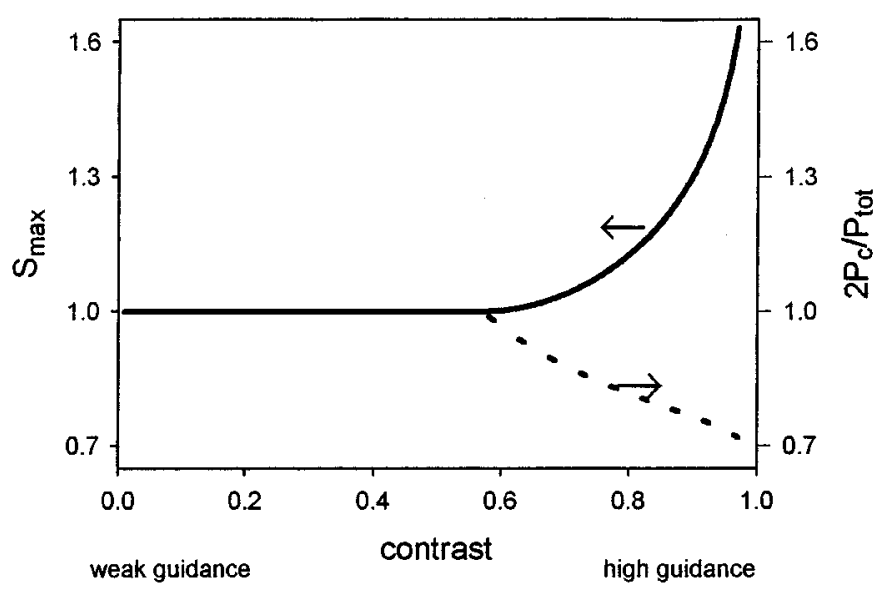

Fig. 2. Maximum achievable sensitivity, $S_{\max }$, (solid line) and the corresponding relative power propagating in the cladding, $2 P_{c} / P_{\text {tot }}$ (dashed line) for the $\mathrm{TM}_{0}$ mode versus the contrast.

with

$$
Y=\frac{1-X}{\alpha_{i}-X}\left[\frac{1+\left(2-\alpha_{i}\right)\left(2 \alpha_{i}-3 X\right)}{1-X\left(2-\alpha_{i}\right)}\right]^{p} \quad i=s, c .
$$

For a given mode-order and polarization, $T$ and $S$ depend only on $X, \alpha_{s}$, and $\alpha_{c}$. Therefore, the optimum normalized effective refractive index, $X_{\text {opt }}$, which ensures the optimum waveguide thickness for maximum sensitivity to changes in the cover for an otherwise fixed set of waveguide parameters, is a function of $\alpha_{s}$ and $\alpha_{c}$ only, and can be found by solving (12). Then, by substituting $X_{\text {opt }}$ in (7) and (2), the maximum sensitivity to changes in the cover, and the corresponding waveguide thickness can now be calculated for every possible combination of $n_{s}, n_{f}, n_{c}$, and $\lambda$.

\section{RESULTS}

The results obtained above can be used for both asymmetric and symmetric waveguides, and it can be shown that the effect, $S>P_{c} / P_{\text {tot }}$, can occur in both cases. Nevertheless, we will limit ourselves to the latter, i.e., $\alpha=\alpha_{s}=\alpha_{c}$, with $\alpha$ being the symmetrical waveguide contrast. In that case the waveguide can be cladded on both sides with the medium to be analyzed, thus improving the sensitivity by a factor of 2 , now $\delta N / \delta n_{\text {cladding }}=\delta N / \delta n_{c}+\delta N / \delta n_{s}=2 \delta N / \delta n_{c}$. For reasons of practical interest, only the results for the $\mathrm{TM}_{0}$ mode will be graphically represented here, although higher order modes show similar behavior. Fig. 2 shows the maximum achievable sensitivity, $S_{\max }=2 \cdot S\left(X_{\mathrm{opt}}\right)$, and the corresponding power propagating in the cladding, $2 P_{c}\left(X_{\mathrm{opt}}\right) / P_{\text {tot }}$, as a function of the contrast $\equiv \alpha$ for the $\mathrm{TM}_{0}$-mode. Fig. 3 shows the corresponding normalized waveguide thickness, $T_{\mathrm{opt}}=T\left(X_{\mathrm{opt}}\right)$. The cutoff condition for the zeroth (i.e., $t=0$ ) and the first-order mode are indicated by the dotted line and can be found, using $N=n_{c}=$ $n_{s}$, from (2) as

$$
T_{\text {cutoff }}=\frac{m \pi}{\sqrt{\alpha}}
$$

As shown in Fig. 2, a region exists where $S_{\max }$ is larger than 1 , which corresponds to the contrast being larger than 0.57 . In this case the waveguide is strictly monomodal and well away

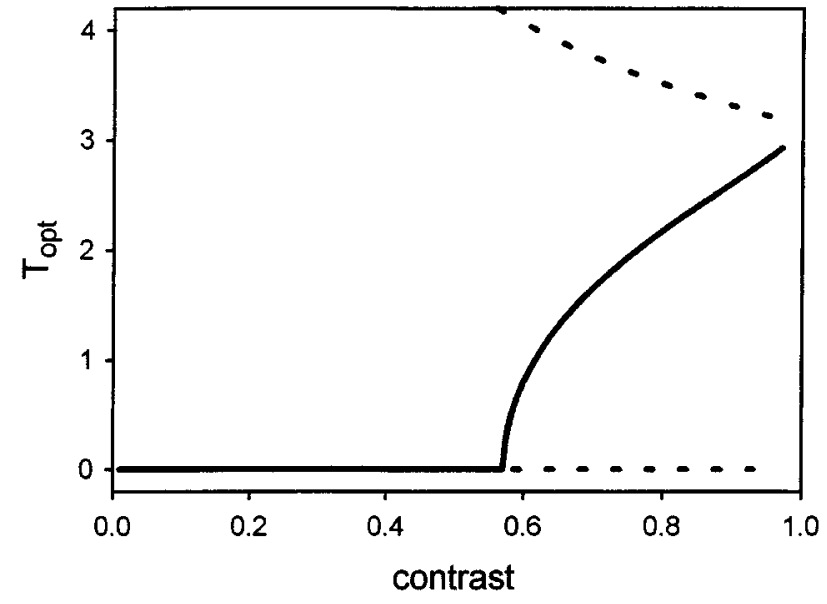

Fig. 3. Normalized waveguide thickness, $T_{\text {opt }}$, ensuring the maximum sensitivity for the $\mathrm{TM}_{0}$ mode versus the contrast. The cut-off condition for the $\mathrm{TM}_{0}$ and the $\mathrm{TM}_{1}$ mode are indicated by dashed lines.

from cutoff (see Fig. 3), while the relative power propagating in the cladding is smaller than 1 . The $S_{\max }$-region where the contrast is smaller than 0.57 corresponds to a maximum sensitivity occurring at the cutoff thickness $(t=0)$ in Fig. 3. This means that symmetric weak guidance waveguides always exhibit a sensitivity smaller than unity, $S=1$ being reached at the cutoff of the $\mathrm{TM}_{0}$ mode, as for the $\mathrm{TE}_{0}$ mode.

It is of major interest to check whether a contrast value larger than 0.57 corresponds to a combination of existing materials. It does indeed since $\alpha>0.57$ corresponds to $n_{f}>1.5 \cdot n_{c}$. This condition can easily be satisfied in the most relevant sensing applications, i.e., where the measurand is a gas, a liquid or an organic film. In the case of a gas, $n_{f}$ must be larger than 1.5 which authorizes the use of practically all slab step-index technologies. In the case of a water cladding, $n_{f}$ must be larger than 2 which is provided by many dielectric films like $\mathrm{Si}_{3} \mathrm{~N}_{4}, \mathrm{ZrO}_{2}$, $\mathrm{HfO}_{2}, \mathrm{Ta}_{2} \mathrm{O}_{5}$ and $\mathrm{TiO}_{2}$. If the cladding material is a high index polymer like polycarbonate $\left(n_{c} \approx 1.6\right) \mathrm{TiO}_{2}$ does fulfill the $S>1$ condition. However, the highest sensitivity will generally be provided by semiconductor films such as Si or GaAs, as shown in the example hereafter.

The formalism and expressions derived here contribute no restriction to the permittivity of the different layers, which may be complex. This means that if the nominal losses of an absorptive sensor are small, which is true in most practical cases, the obtained maximum sensitivities for a lossless structure also hold for a change in the imaginary part of the refractive index. This implies that absorption enhancement can be obtained. As an example of this, an absorption sensor for detecting gas concentrations at a specific absorption line [9] will be proposed.

A freely suspended silicon waveguide $\left(n_{f}=3.45\right)$ in a gas atmosphere $\left(n_{\text {cladding }}=1\right)$ has a contrast, $\alpha=0.916$. A wavelength of $\lambda=1550 \mathrm{~nm}$ is assumed. Then, from Figs. 2 and 3 , and using (6), a sensitivity $S_{\max }=1.35$ can be obtained for a waveguide thickness of $t=192 \mathrm{~nm}$. This means that although only $76 \%$ of the guided power is located in the cladding (see Fig. 2), the absorption of the light by the gas is enhanced by a factor 1.35 compared to that of a free-space beam propagating in the same gas. This implies that an integrated optical 
read-out and sensing circuit can be used with an even higher sensitivity than in the free-space configuration. This result was checked by calculating the mode attenuation with a commercially available mode solver [11] and with the expressions for the sensitivity given in [4]. In addition it was independently confirmed by calculating the propagation of a Gaussian light distribution into the discussed structure using a commercially available BPM-algorithm; in that way avoiding the use of modal expansions [11]. Note that absorption changes in an aqueous cladding $\left(n_{c} \approx 1.33\right)$ combined with a silicon waveguide will also lead to absorption enhancement $(S=1.2)$.

Since gain in a medium can simply be seen as negative absorption, the obtained results might also be relevant for obtaining larger optical amplification. For example the gain in an erbium-doped silica waveguide $(n \approx 1.45, \lambda=1550 \mathrm{~nm}$ ) can be enhanced by a factor of 1.16 , by using a silicon waveguide cladded with erbium-doped silica instead $(t=162 \mathrm{~nm})$.

\section{PHYSICAL EXPLANATION}

The physical explanation of the enhanced absorption is simple if one realizes that the absorption rate at a given position $\boldsymbol{r}$ is proportional to $|\boldsymbol{E}(\boldsymbol{r})|^{2}$ where $\boldsymbol{E}(\boldsymbol{r})$ is the electrical field at the position $r$.

Now we have to compare the interaction of a plane wave in a homogeneous material with absorption and a refractive index $n_{c}$, with the interaction of a TM mode of which only the evanescent waves propagate through that material. Both systems differ with respect to the part of the power propagating through the absorptive material, but also in the propagation speed and the direction of the magnetic field factor. The first aspect leads, without any doubt to a lowering of the attenuation, with a factor of $P_{c} / P_{\text {tot }}$. Both other factors will be shown to lead to an enhancement of the attenuation.

In the first place, at a given power the amount of energy in a slice perpendicular to the channel axis and a slice thickness of one unit length is proportional to the inverse of the propagation speed of the guided beam, implying that it is proportional to effective refractive index $N$. Hence, compared to the free-space beam we win (because $N>n_{c}$ ) a factor $N / n_{c}$, emphasizing the importance of having a high index of the core layer.

In the second place, at a position $\boldsymbol{r}$ that absorption rate is proportional to $|\boldsymbol{E}(\boldsymbol{r})|^{2}$. For the free-space beam, just as for TE-modes the E-field consists of the lateral field component $E_{y}$ only, in TM-modes, however, there are two components: a transversal one, $E_{x}$ and another one being parallel to the propagation direction, $E_{z}$.

This implies that in the case of a TM mode the absorption rate at a given position $\boldsymbol{r}$ is proportional to

$$
|\boldsymbol{E}(\boldsymbol{r})|^{2}=\left|E_{x}(\boldsymbol{r})\right|^{2}+\left|E_{z}(\boldsymbol{r})\right|^{2} .
$$

The transmitted power however, given as the Poynting vector only depends on the lateral field component $E_{x}(\boldsymbol{r})$ being proportional to $E_{x}(\boldsymbol{r})^{2}$ (see Appendix). So the $E_{z}$ component of the field is active in the absorption, but does not manifest itself in the transmitted power. As a consequence a second factor enhancing the absorption at points in the cladding region arises, this factor being specific for TM modes

$$
\left\{\left|E_{x}(\boldsymbol{r})\right|^{2}+\left|E_{z}(\boldsymbol{r})\right|^{2}\right\} /\left|E_{x}(\boldsymbol{r})\right|^{2} .
$$

Taking in account, that absorption takes place in the cladding region of the symmetrical three layer slabguide only, this enhancement factor can be expressed analytically by substituting the well known relations between the fields $E_{x}, E_{z}$, and $H_{y}$ (see also Appendix). This leads to

$$
\left\{\left|E_{x}(\boldsymbol{r})\right|^{2}+\left|E_{z}(\boldsymbol{r})\right|^{2}\right\} /\left|E_{x}(\boldsymbol{r})\right|^{2}=1+\left(N^{2}-n_{c}^{2}\right) / N^{2} .
$$

Combining all factors, it can be concluded that by launching identical powers into a free-space beam, propagating through an absorbing medium and into a TM mode of a symmetrical three layer slabguide where the cladding consists of the same absorbing medium, the attenuation of the waveguiding system is enhanced relative to that of a free-space beam with a factor

$$
S=N / n_{c} \cdot\left\{1+\left(N^{2}-n_{c}^{2}\right) / N^{2}\right\} \cdot P_{c} / P_{\text {tot }} .
$$

The relation again shows the importance of a high value of the modal index $N$, although there has to be made a compromise with respect to obtaining also a high fraction of the power propagating through the cladding region. The latter is also favored by choosing a low value of the thickness of the core layer.

Note, that in this calculations we have not taken into account the influence of the absorption coefficient on the modal field profile. The latter however can be calculated to be negligible. Also, it has to be noted, that the same relation can be found by substituting relations (4) and (5) into relation (7), but now by starting from more basic physics, the physical background of this formula has been made clear.

The same type of reasoning holds for real changes in refractive index of the cladding material.

The physical explanation of the enhancement effect can also be demonstrated by perturbation theory (see Appendix).

\section{CONCLUSION}

We have shown that in the case of homogeneous sensing with a three-layer slab waveguide the sensitivity of the effective refractive index to variations of the cladding index can be larger than unity for TM polarization and strong guidance. The observation is counter intuitive but can be explained when the differences in power fraction in the absorptive material, in the propagation speed and in the direction of the magnetic field factor of both systems are considered. Although the enhancement might be too small to compensate for the difficulty of injecting light into a waveguide, it opens up new development perspectives for chemical sensors within the emerging field of silicon based microsystems as well as for integrated optical amplifiers. It can be shown, using simple arguments from integrated optics theory, that the presented effect may even be larger in large contrast free standing channel waveguides, like beams, stripes and circular waveguides, if well optimized. 


\section{APPENDIX}

The origin of the sensitivity enhancement can clearly be seen from perturbation theory. Here the absorption or the change in refractive index is considered to be a perturbation on the not-absorbing or unchanged system. Perturbation theory presents the change of the propagation constant as a function of the change of the complex refractive index in a certain region, in this special case in the cladding material.

Perturbation theory [12] gives us for the zeroth-order TM-mode the relation

$$
\Delta \beta^{2}=\int \frac{H_{y}}{\varepsilon_{x}} \Delta O H_{y}^{*} d x / C_{0}
$$

where $O H_{y}$ is defined by the eigenvalue equation for TM polarized light in planar structures

$$
O H_{y} \equiv \varepsilon_{x} \partial_{x}\left(\frac{1}{\varepsilon_{z}} \partial_{x} H_{y}\right)+k_{0}^{2} \varepsilon_{x} H_{y}=k_{0}^{2} N^{2} H_{y} \equiv \beta^{2} H_{y}
$$

where $\beta \equiv k_{0} N$ and $C_{0}$ is defined by the orthogonality theorem [10]

$$
\int H_{y}^{p} H_{y}^{q^{*}} / \varepsilon_{x} d x=\delta_{p q} C_{p}=\delta_{p q} P_{p} 2 \omega \varepsilon_{0} / \beta
$$

where $p(=0)$ and $q$ indicate the mode order.

We assume a small (isotropic) and uniform perturbation in (part of) the layers, but for clarity we define an individual $\Delta \varepsilon_{z}$ and $\Delta \varepsilon_{x}$ according to

$$
\varepsilon_{c} \rightarrow \varepsilon_{c}+\Delta \varepsilon_{c} ; \Delta \varepsilon_{c}=\Delta \varepsilon_{x}=\Delta \varepsilon_{z} .
$$

Further elaboration of formula (A1), then gives the result

$\Delta \beta^{2}=\int\left\{\frac{\Delta \varepsilon_{z}}{\varepsilon_{z}^{2}}\left|\partial_{x} H_{y}\right|^{2}+\beta^{2} \frac{\Delta \varepsilon_{x}}{\varepsilon_{x}^{2}}\left|H_{y}^{2}\right| d x\right\} / C_{0}$.

Equation (A5) can be rewritten as

$$
\begin{aligned}
\Delta \beta^{2}= & \left\{\int H_{y} \frac{\varepsilon_{x}+\Delta \varepsilon_{x}}{\varepsilon_{x}} \partial_{x}\left(\frac{1}{\varepsilon_{z}+\Delta \varepsilon_{z}} \partial_{x} H_{y}^{*}\right) d x\right. \\
& -\int H_{y} \partial_{x}\left(\frac{1}{\varepsilon_{z}} \partial_{x} H_{y}^{*}\right) d x \\
& \left.+\int k_{0}^{2} \Delta \varepsilon_{x}\left|H_{y}^{2}\right| / \varepsilon_{x} \cdot d x\right\} / C_{0} .
\end{aligned}
$$

Hence, after some simple manipulations

$$
\begin{aligned}
\Delta \beta^{2}= & \left\{\int H_{y} \partial_{x}\left(-\frac{\Delta \varepsilon_{z}}{\varepsilon_{z}^{2}} \partial_{x} H_{y}^{*}\right) d x\right. \\
& +\int \frac{\Delta \varepsilon_{x}}{\varepsilon_{x}} H_{y} \partial_{x}\left(\frac{1}{\varepsilon_{z}} \partial_{x} H_{y}^{*}\right) d x \\
& \left.+k_{0}^{2} \int \Delta \varepsilon_{x}\left|H_{y}^{2}\right| / \varepsilon_{x} d x\right\} / C_{0} .
\end{aligned}
$$

The first integral can be rewritten by integration by parts, for the second and third integrals we use the modal field equation leading to

$$
\Delta \beta^{2}=\int\left\{\frac{\Delta \varepsilon_{z}}{\varepsilon_{z}^{2}}\left|\partial_{x} H^{y}\right|^{2}+\beta^{2} \frac{\Delta \varepsilon_{x}}{\varepsilon_{x}^{2}}\left|H_{y}^{2}\right| d x\right\} / C_{0} .
$$

So, from (A8) it can be seen that both $\Delta \varepsilon_{x}$ and $\Delta \varepsilon_{z}$ (and so both electric field components) contribute to the modal index changes.

Taking into account the relations between the two electric field components and $H_{y}$

$$
E_{x}=\beta H_{y} /\left(\omega \varepsilon_{0} \varepsilon_{x}\right)
$$

$$
E_{z}=-i \partial_{x} H_{y} /\left(\omega \varepsilon_{0} \varepsilon_{z}\right)
$$

relation (A8) can be transformed into

$$
\Delta \beta^{2}=\beta^{2} \int d x\left\{\Delta \varepsilon_{z}\left|E_{z}\right|^{2}+\Delta \varepsilon_{x}\left|E_{x}\right|^{2} /\left(\varepsilon_{x}\left|E_{x}\right|^{2}\right)\right\} .
$$

Setting $\Delta \varepsilon_{x}=\Delta \varepsilon_{z}=\Delta \varepsilon_{c}$ the relation can be rewritten as

$$
\Delta \beta^{2}=\beta^{2} \Delta \varepsilon / \varepsilon_{c} \int_{c} d x\left\{\left(\left|E_{x}\right|^{2}+\left|E_{z}\right|^{2}\right) /\left|E_{x}\right|^{2}\right\}
$$

in which we recognize the enhancement factor given in relation (16). In (A12), the subscript $c$ indicates that the integration is over the two outermost layers. From (A12) it follows:

$$
k_{0}^{2} 2 N \Delta N=\left\{2 \Delta n_{c} k_{0}^{2}\left(N^{2}-n_{c}^{2}\right)+k_{0}^{2} 2 \Delta n_{c}\right\} P_{c} /\left(n_{c} P_{\text {tot }}\right) .
$$

Here, we have used that $\partial_{x} H^{y}= \pm \sqrt{N_{2}-n_{c}^{2}} k_{0} H_{y}$ in the outermost layers, and

$$
P_{c} / P_{\text {tot }} \equiv \int_{c}\left|H_{y}\right|^{2} / \varepsilon_{c} \cdot d x / \int\left|H_{y}\right|^{2} / \varepsilon_{x} \cdot d x .
$$

From (A13) it follows for the sensitivity:

$$
S \equiv \frac{\partial N}{\partial n_{c}}=\frac{N}{n_{c}}\left\{1+\left(N^{2}-n_{c}^{2}\right) / N^{2}\right\} P_{c} / P_{\text {tot }}
$$

just the same relation as we derived earlier in Section IV.

Analytical expressions for $P_{c} / P_{\text {tot }}$ can be found from standard theory [4] leading to

$$
\begin{aligned}
P_{c} / P_{\text {tot }}=\{1+\cos (\gamma t)\} \varepsilon_{g} \gamma /[ & \left\{(1+\cos (\gamma t)\} \varepsilon_{g} \gamma\right. \\
& \left.+\{\gamma t+\sin (\gamma t)\} \varepsilon_{c} \kappa\right]
\end{aligned}
$$

with

$$
\gamma \equiv k_{0} \sqrt{n_{g}^{2}-N^{2}}
$$

and

$$
\kappa \equiv k_{0} \sqrt{N^{2}-n_{c}^{2}}
$$

In the above, $t$ is the thickness of the guiding layer.

\section{REFERENCES}

[1] R. G. Heideman, G. J. Veldhuis, E. W. H. Jager, and P. V. Lambeck, "Fabrication and packaging of integrated chemo-optical sensors," Sensors Actuators, vol. B35-36, pp. 234-240, 1996. 
[2] B. J. Luff, J. S. Wilkinson, J. Piehler, U. Hollenbach, J. Ingenhoff, and N. Fabricius, "Integrated optial Mach-Zehnder biosensor," J. Lightwave Technol., vol. 16, pp. 583-592, 1998.

[3] A. Bernard and H. R. Bosshard, "Real-time monitoring of antigen-antibody recognition on a metal oxide surface by an optical grating coupler sensor," Eur. J. Biochem., vol. 230, pp. 416-423, 1995.

[4] K. Tiefenthaler and W. Lukosz, "Sensitivity of grating couplers as integrated-optical chemical sensors," J. Opt. Soc. Amer. B, vol. 6, pp. 209-220, 1989.

[5] G. J. Veldhuis, J. H. Berends, R. G. Heideman, and P. V. Lambeck, "An integrated optical Bragg-reflector used as chemo-optical sensor," Pure Appl. Opt., pp. L23-L26, 1998.

[6] G. J. Veldhuis and P. V. Lambeck, "Highly-sensitive passive integrated optical spiral-shaped waveguide refractometer," Appl. Phys. Lett., vol. 71, pp. 2895-2897, 1997.

[7] A. Bradenburg and R. Edelhäuser, "Integrated optical gas sensors using organically modified silicates as sensitive films," Sensors Actuators B, vol. 11, pp. 361-374, 1993

[8] O. Parriaux and G. J. Veldhuis, "Normalized analysis for the sensitivity optimization of integrated optical evanescent wave sensors," J. Lightwave Technol., vol. 16, pp. 573-582, 1998.

[9] K. Chan, H. Ito, and H. Inaba, "An optical-fiber-based gas sensor for remote absorption measurement of low-level $\mathrm{CH}_{4}$ gas in the near-infrared region," J. Lightwave Technol., vol. LT-2, pp. 234-237, 1984.

[10] H. Kogelnik and T. Tamir, Topics in Applied Physics. New York: Springer-Verlag, 1975, vol. 7, ch. 2.

[11] BBV Software B. V., Enschede, The Netherlands.

[12] L. I. Schiff, Quantum Mechanics. New York: McGraw-Hill, 1955, ch. 7, pp. 151-155.
G. J. Veldhuis, photograph and biography not available at the time of publication.

O. Parriaux, photograph and biography not available at the time of publication.

H. J. W. M. Hoekstra, photograph and biography not available at the time of publication.

P. V. Lambeck, photograph and biography not available at the time of publication. 